\title{
ASSOCIATION BETWEEN LIPID LEVEL AND CHRONIC PERIODONTITIS IN PRODUCTIVE AGE
}

\author{
Bonita Putri Arinida, Adik Wibowo \\ Department of Health Policy and Administration, Faculty of Public Health, \\ Universitas Indonesia
}

\begin{abstract}
Background: Periodontitis affects at least $72 \%$ of Indonesian community according to Indonesian Basic Health Research (Riskesdas) in 2018. Periodontitis is considereded as localized inflammation. However, the condition leads to systemic change. The recognition of systemic change was observed in lipid levels by several earlier studies. This study aimed to examine the association between lipid level and chronic periodontitis and healthy periodontal status in productive age.

Subjects and Method: This was a cross-sectional study conducted in a company clinic, in 2019. A total of 120 patients were selected consisting of 60 patients diagnosed with chronic periodontitis and the 60 patients with healthy periodontal status. The data of periodontitis patients were obtained from medical check-up. Data of lipid levels included total cholesterol, triglycerides, low-density lipoprotein (LDL) cholesterol, and high-density lipoprotein (HDL) cholesterol. The data were analyzed by a multiple linear regression.

Results: Patients with chronic periodontitis showed higher LDL cholesterol $(\mathrm{p}<0.001)$ and triglycerides $(\mathrm{p}=\mathrm{O} .003)$ than patients with normal periodontal status. Total cholesterol was higher in patients with chronic periodontitis than healthy periodontal status ( $p<0.001)$. The difference in HDL cholesterol was statistically non-significant between patients with chronic periodontitis and healthy periodontal status $(\mathrm{p}=0.963)$.

Conclusion: Lipid level can be an indicator of periodontal disease and give more information to perform higher quality of examination. However, more studies are needed to strengthen the evidence for improving the medical check-up coverage.
\end{abstract}

Keywords: periodontitis, lipid level, inflammation, diagnoses

\section{Correspondence:}

Bonita Putri Arinida. Department of Health Policy and Administration, Faculty of Public Health, Universitas Indonesia, Depok West Java. Email: bonitaarinidadrg@gmail.com. Mobile: 081282269019. 